Gesamturteil am Maßstab des $\$ 7$ II AtomG sowie eine formelle Öffentlichkeitsbe-

teiligung erfolgen müssen. Dieses "formelle» Entscheidungsverfahren konnte nicht durch nachträgliche Anordnungen oder durch eine Zusicherung auf Nichteinschreiten umgangen werden.

\title{
VI. Ausblick
}

Die bisherige juristische Auseinandersetzung spielte sich schwerpunktmäßig auf strafrechtlicher Ebene ab. Es entsteht der Eindruck, als ob die verwaltungsgerichtliche Kontrolle von vornherein versagt. Jedoch auch das strafrechtliche Vorgehen änderte an der sozialen Wirklichkeit nichts. Das Landgericht Hanau und das überwiegende strafrechtliche Schrifttum haben im Ergebnis zu recht eine Bestrafung abgelehnt; die Vorabzustimmungen nicht nur als rechtswidrig, sondern sogar als nichtig zu qualifizieren, vermag nicht besonders zu überzeugen: Hier kann man nur mit Evidenz-Leerformeln argumentieren.

Der Presse war zu entnehmen, daß der zuständige Minister überprüft hat, welche Konsequenzen für die Vorabzustimmungen aus dem Hanauer Urteil zu ziehen seien. Dolde hebt zutreffend hervor, daß die strafrichterliche Würdigung die Verwaltung nicht binden $\mathrm{kann}^{91}$. Teilweise wurden die Bescheide in Teilgenehmigungen umgewandelt. Betroffene Dritte könnten in dieser Lage Anfechtungsklage zumindest gegen die umgewandelten Bescheide erheben ${ }^{92}$; diesen Klagen käme aufschiebende Wirkung zu, so daß der aus diesen Bescheiden abgeleitete Betrieb rechtswidrig wäre. Gegenüber der Anordnung einer sofortigen Vollziehung bestünde die Möglichkeit vorläufigen Rechtsschutzes.

Ebenso könnte man in Betracht ziehen, gegen bestandskräftige Vorabzustimmungen vorzugehen. Durch die erneute Überprüfung der Bescheide hat der Minister die Verwaltungsverfahren von Amts wegen wieder aufgenommen. Dritte könnten versuchen, in diesen Verwaltungsverfahren beteiligt zu werden und eine Aufhebung der rechtswidrigen Bescheide zu erreichen. Gegebenenfalls könnte nach $\mathbb{\$} \mathrm{I}$ HVwVfG die Wiederaufnahme des Verfahrens beantragt werden. Sollte durch die aufschiebende Wirkung oder die Aufhebung von Bescheiden die Betriebsgrundlage für die Nuklearbetriebe entfallen, könnten Dritte versuchen, die Behörde zur vorläufigen Stillegung auf Grundlage des $\$ 19$ III AtomG zu verpflichten. Theoretisch bestehen also einige Wege, die Verwaltungsgerichtsbarkeit zur Realisierung ihres Kontrollauftrages zu veranlassen.

\section{Klaus Sieveking Ausländerpolitische Zwecke im Sozialrecht}

Neuere Tendenzen der Verpolizeilichung des Sozialrechts

Es gehört zu den Erfahrungen des letzten Jahrhunderts, daß mit dem Anwachsen der Freizügigkeit der Bürger der Zusammenhang zwischen dem Recht auf Armenfürsorge und Heimatprinzip zunehmend funktionslos wird. Die Sozialpolitik entwickelte sich seit 1794 (der Geltung des Allgemeinen preußischen Landrechts) als

9r Dolde (Anm. 8), NJW 88, 2329 (2336), Anm. 6.

92 Ein Widerspruchsverfahren findet nach $\$ 68$ I 2 Nr. I VwGO nicht statt. 
eine Staatsaufgabe, deren ordnende und leistende Gestaltung nicht nur auf »Untertanen «, sondern auch auf alle Ortsansässigen (Wohnsitzprinzip) gerichtet war. Das Fehlen (oder die Entziehung) des aktiven und passiven Wahlrechts bei Inanspruchnahme der Armenunterstützung ${ }^{1}$ war der politische Ausdruck einer Koppelung von polizeilichen mit sozialen Zwecken und zugleich Ausdruck der staatspolitischen Diskriminierung von Bürgern, die über kein »ordentliches A Arbeitseinkommen verfügten.

Die ordnungspolitische Zweckverfolgung im Rahmen einer sozialrechtlichen Entscheidung steht im Zentrum dieses Beitrages. Diese Betrachtungsweise knüpft an Überlegungen an, die sich auf die Debatte über Verrechtlichung im Bereich des Sozialrechts beziehen ${ }^{2}$ und die den Zusammenhang des Sozialrechts mit Krisenentwicklungen verdeutlicht und das Sozialrecht als integralen Bestandteil staatlicher Globalsteuerung gekennzeichnet haben ${ }^{3}$. In einer anderen Erscheinungsform kann dieser Tatbestand nämlich heute wieder (noch?) identifiziert werden. Da sich Polizeirecht und Sozialrecht in formalrechtlicher Hinsicht historisch weiter verselbständigt und auseinanderentwickelt haben, interessiert die aktuelle inhaltliche Verknüpfung dieser Bereiche ${ }^{4}$. Den folgenden Ausführungen liegt die These zugrunde, $\mathrm{daß}$ das Sozialrecht ordnungspolitische und polizeiliche Zwecke in sich aufnimmt, ohne daß damit die Funktion des Sozialrechts, defizitäre Soziallagen auszugleichen oder zu beseitigen, verfolgt wird. Die hier näher beschriebenen Beispiele beziehen sich auf eine Erscheinungsform dieser Tendenz, bei der die frühere fremdenpolizeiliche Rolle der Fürsorge in dem Gewande sozialpolitischer Entscheidungen ("Rückkehrförderung ") erneut wirksam wird: Sie nehmen die Tatsache der zunehmenden Ausgrenzung von Ausländern aus Nicht-EG-Staaten vom deutschen Arbeitsmarkt und dem deutschen Sozialleistungssystem in den Blick. Diese Politik, die ihre Legitimation in sozialrechtlichen Normen findet, hat nämlich nicht nur die Gefährdung, sondern teilweise auch den Verlust des Aufenthaltsrechts der Ausländer zur Folge. Angesichts der heute verstärkt sichtbaren Internationalisierung der Arbeitsmärkte erhalten die hier angesprochenen Erscheinungsformen der Koppelung von Ausländer- und Sozialpolitik/-rechts eine erhebliche gesellschaftspolitische Bedeutung.

\section{Die ausländerpolitische Ausgangslage}

Im Zentrum der folgenden Bemerkungen steht die Lage von Staatsangehörigen aus Staaten außerhalb der Europäischen Gemeinschaft (Drittstaater). Ihnen gegenüber haben sich Politik, Recht und soziale Sicherung in den vorangegangenen Jahren immer wieder verändert - mit im wesentlichen von den wirtschaftlichen, später eher von finanz- und gesellschaftspolitischen Bedürfnissen bestimmten Akzentuierungen.

1 Zur sozialethischen und politıschen Diskrımınerung der Armen siehe M. Stollets, Quellen zur Geschichte des Sozialrechts, Göttıngen 1976, S. 19 mit Nachwersen in Fußnote 26.

2 Hierzu ausführlich H.-F. Zacher, Verrechtlichung im Bereich des Sozıalrechts, in: F. Kübler (Hrsg.), Verrechtlichung von Wirtschaft, Arbeit und sozıaler Solidarität, Baden-Baden ig84, S. I Iff.

3 Vgl. U. Mückenberger, Thesen zur Funktion und Entwicklung des Sozialrechts, KJ 1976, S. $34 \mathrm{Iff}$. (349ff.).

4 Zum Zusammenhang von Fürsorge und Armenpolizeı sıehe F. Barabas/Chr. Sachße, Bundessozıalhilfegesetz: Sozialstaatliche Versorgung oder Armenpolizei?, K] 1976, S. $359 \mathrm{ff}$.

5 Siehe allgemen dazu: Die sozialrechtliche Stellung der Ausländer in der Bundesrepublik Deutschland, Wiesbaden 1983. K. M. Groth/J.Müller-Gazurek, Ausländer - Sozialrecht, Frankfurt/Main 1983; K. Barwıg/K. Lörcher/Chr. Schumacher (Hrsg.), Soziale Sicherung und Aufenthaltsrecht, Baden-Baden 1986. 
Die Ausländerrechtspolitik gegenüber Drittstaatern läßt sich aus heutiger Sicht in mehrere historische Phasen einteilen, die wie folgt zu charakterisieren sind:

- die Ausländeranwerbung (I955 bis 1973),

- die Zuzugsbegrenzung bei gleichzeitig zögerlicher Integration und zunehmender Ausgrenzung der hier lebenden Ausländer aus dem Sozialleistungssystem (1973 bis 1982),

- die Rückkehrförderung bei gleichzeitiger Steuerung des Familiennachzugs (1982 bis 1986),

- die bis heute anhaltende Familiennachzugskontrolle, die faktısche Zurückdrängung durch sozialpolitische Auszehrung und das Anwachsen von Ausländerfeindlichkeit bei gleichzeitig zunehmender kontingentierter Zulassung ausländischer Arbeitskräfte als sogenannte Werkvertragsarbeitnehmer (vornehmlich Türken und Jugoslawen nach Spezialbedarf in einzelnen Wirtschaftsbereichen) und anwachsender Aussiedlerströme aus dem osteuropäischen Raum.

Sozialpolitik und Sozialrecht werden erkennbar erst in der zweiten Phase als Mittel der Ausgrenzung von Ausländern aus dem deutschen Arbeitsmarkt und dem deutschen Sozialleistungssystem eingesetzt. Als Beleg für diese These ließen sich zahlreiche Beispiele benennen. Hier sollen - korrespondierend zu einzelnen oben genannten Phasen - lediglich drei typische Beispiele herausgegriffen und beschrieben werden. Sie beziehen sich auf die rechtspolitischen Aktionsfelder Rechtsprechung, Gesetzgebung und Verwaltung, innerhalb deren Wirkungsbereich Mittel der Ausgrenzung und Rechtfertigungsstrategien für ausländerspezifische Diskriminierungen entwickelt werden.

\section{Die Ausgrenzung arbeitsloser Ausländer aus dem Leistungssystem der Arbeitslosenversicherung}

Das erste Beispiel betrifft die Ausgrenzung jener arbeitslosen Ausländer aus dem Leistungssystem der Arbeitslosenversicherung, die - anders als EG-Staatsangehörige - keinen freien Zugang zum Arbeitsmarkt der Bundesrepublik Deutschland haben; betroffen sind vor allem türkische und jugoslawische Arbeitnehmer. Aufgrund der Rechtsprechung des Bundessozialgerichts ${ }^{6}$ werden den arbeitslosen ausländischen Arbeitnehmern ohne besondere Arbeitserlaubnis ${ }^{7}$ Ansprüche auf $\mathrm{Ar}$ beitslosenhilfe entzogen, wenn ihnen der bundesdeutsche Arbeitsmarkt verschlossen ist. Dies soll im wesentlichen dann der Fall sein, wenn ein Jahr lang (während des Bezugs von Arbeitslosengeld oder -hilfe) erfolglos versucht wurde, sie in eine geeignete Beschäftigung $\mathrm{zu}$ vermitteln oder eine passende Fortbildungs- bzw. Umschulungsmaßnahme zu finden. Hierbei ist zu berücksichtigen, daß diese ausländischen Arbeitnehmer gegenüber Deutschen, EG-Ausländern und Inhabern einer besonderen Arbeitserlaubnis nach der Praxis der Bundesanstalt für Arbeit bereits nachrangig vermittelt werden (»Vorrangprinzip«). Folge dieser Praxis ist es, $\mathrm{da} ß$ arbeitslose Ausländer, die nach einem Jahr als nicht vermittelbar angesehen werden, der Arbeitsverwaltung nicht mehr zur Verfügung stehen. Dies führt zur Ausgrenzung aus dem Leistungssystem des Arbeitsförderungsgesetzes und zum Abdrängen in die Sozialhilfe - mit allen Folgen der Verunsicherung wegen möglicher Ausweisung ${ }^{8}$.

Die Besonderheit dieses Falles von arbeitsförderungsrechtlicher Ausgrenzung be-

6 BSG NJW 1978, S. 1 i 25 (Urteil vom 27. 1. 1977) = SGb 1978, S. 30 s mit Anmerkung von M. Wollenschläger.

7 Im Falle des Besıtzes der besonderen Arbeıtserlaubnis sind Ausländer den Deutschen und sonstıgen EGStaatsangehörıgen gleıchgestellt. Zum Arbeitserlaubnisrecht siehe Urteil des BSG vom 23.6.1982, SGb ${ }_{1983}$, S. 404 mit Besprechungsaufsatz von G. Schwerdtfeger, SGb 1983, S. 373 . Allgemein K.-J. Bieback, Arbeitserlaubnisrecht, Frankfurt/Main 1985 .

8 Zu weıteren Einschränkungen der sozialen Sicherung durch Statusgefährdung sıehe K. Sievekıng, Soziale Sicherung für Ausländer, Nachrichtendienst des Deutschen Vereıns für öffentliche und private Fürsorge (NDV) 1988, S. 36, (37ff.) 
steht in der Steuerungsmethode: Das Kriterium des verschlossenen Arbeitsmarktes wird - methodisch höchst fragwürdig, wenn nicht unzulässig - ohne normativen Anhaltspunkt als ungeschriebenes Tatbestandsmerkmal der »Verfügbarkeit « eingeführt. Die Tatsache der Verschlossenheit des Arbeitsmarktes wird faktisch von der Verwaltungspraxis der Arbeitsverwaltung festgelegt. Die Bundesanstalt für Arbeit hat - ebenso wie bei dem Vorrangprinzip - in zahlreichen Verwaltungsvorschriften (Runderlasse, Dienstanweisungen) den Dienststellen der Arbeitsvermittlung entsprechende Entscheidungsorientierungen an die Hand gegeben?. Sie hat damit der Ausgrenzung von ausländischen Arbeitnehmern aus dem deutschen Arbeitsmarkt Vorschub geleistet und ist letztlich Garant der damit bezweckten ausländerpolitischen Ziele.

\section{Die Gesetzgebung zur Förderung der Rückkehrbereitschaft von Ausländern $1983 / 84$}

Als weiteres Beispiel ist vor allem die Rückkehrförderungsgesetzgebung zu erwähnen. Mit dieser Gesetzgebung im Rahmen der sog. Rückkehrförderungspolitik waren seinerzeit sozialrechtliche Ansprüche verändert beziehungsweise gekürzt worden, um dadurch ausländerpolitische Ziele zu erreichen. Mit dem »Gesetz zur Förderung der Rückkehrbereitschaft von Ausländern $\aleph^{10}$ hat man vordergründig Ansprüche gewährt (sog. Rückkehrhilfen und die vorzeitige Kapitalisierung von Rentenversicherungsansprüchen ohne die sonst übliche Wartefrist von zwei Jahren im Rahmen der Beitragserstattung); bezweckt war allerdings eine rechtlich legitimierte (zulässige und abgesicherte) Verringerung der Arbeitslosen insbesondere durch Ausgrenzung der Ausländer vom deutschen Arbeitsmarkt ${ }^{11}$. Mit Blick auf die Tendenz der Verpolizeilichung des Sozialrechts wird man dieses Beispiel allerdings nur in einer vermittelten und deshalb abgeschwächten Form gelten lassen können. Angesichts des völkerrechtlichen Verbots von Massenausweisungen ${ }^{\text {I2 }}$ stellt sich die Frage nach dem polizeilichen Charakter materieller Anreize zur freiwilligen Ausreise unter dem Blickwinkel eines zulässigen Ersatzes zu ordnungspolitischen Zwecken - insoweit kann das Beispiel der Rückkehrförderungsgesetzgebung nur in eingeschränkter, aber dennoch anschaulicher Weise Tendenzen verdeutlichen.

Der »Anreiz«, das Gebiet der Bundesrepublik Deutschland zu verlassen, bestand zum einen in der Auszahlung von Geld in Form der Rückkehrhilfe (»Rückkehrprämie «). Der in Aussicht gestellte Betrag hing seinem Umfang nach von dem Zeitpunkt ab, innerhalb dessen Betroffene nach Eintritt der Arbeitslosigkeit die Bundesrepublik auf Dauer verließen. Prämiert wurde der schnell durchgeführte Entschluß zur Rückkehr (was Mitnahmeeffekte der schon früher zur Rückkehr Entschlossenen ermöglichte) $)^{13}$. Zum anderen erleichterte der Sozialgesetzgeber den in diesem Zeitraum ausreisewilligen Arbeitnehmern die Kapitalisierung erworbener Renten-

\footnotetext{
9 Siehe die Dienstanwersung zum Arbeitserlaubnısrecht (DA AER), Stand April 1987. Die vorangegangene Fassung der DA AER ist veröffentlicht bel K. Sieveking, Sammlung von Rechtsvorschriften für Ausländer im Land Bremen, Heft s (Stand: 31. 12. 1985), ZERP MAT' 10, S. $123 \mathrm{ff}$.

II Zur Problematik der Rückkehr als wırtschaftliches und sozıales Risiko E. Hönekopp (Hrsg.), Rückkehrförderung und Rückkehr ausländischer Arbeitnehmer - Ergebnısse des Rückkehrförderungsgesetzes, der Rückkehrhilfe-Statistik und der IAB-Rückkehrerbefragung, in: ders. (Hrsg.), Aspekte der Ausländerbeschäftıgung in der Bundesrepublik Deutschland, Nürnberg 1987 (Beıträge zur Arbettsmarkt- und Berufsforschung I I4), S. $287 \mathrm{ff}$. und $R$. Votgt (Hrsg.), Gastarbeiter zwischen Integration und Remigratıon, HiMoN-Sammelband S 3 , Siegen 1986. Siehe auch H.Körner/U. Mebrländer (Hrsg.), Die »neue* Ausländerpolitik un Europa. Erfahrungen in den Aufnahme- und Entsendeländern, Bonn 1986.

12 Vgl. Art. 4 des 4 .Zusatzprotokolls zur Europäischen Menschenrechtskonvention.

$13 \mathrm{Vgl}$. Hönekopp (Fn I I), S. $287 \mathrm{ff}$.
} 
anwartschaften in Form der Beitragserstattung, ohne daß die sonst übliche Wartefrist von zwei Jahren galt; von dieser vorzeitigen Beitragserstattung konnten auch diejenigen berechtigten Ausländer Gebrauch machen, deren Wartefrist schon zu laufen begonnen hatte, d. h. die das Bundesgebiet schon vorher verlassen hatten ${ }^{14}$.

Mit dem Rückkehrförderungesetz war eine deutliche Entlastung des deutschen Arbeitsmarktes von arbeitslosen Ausländern - insbesondere von türkischen Arbeitnehmern - bezweckt ${ }^{15}$. Mit dem "Anreiz« der vorzeitigen Beitragserstattung, die insbesondere Türken und Portugiesen (aufgrund des damals geltenden internationalen Abkommensrechts) anstelle einer späteren deutschen Altersrente wählen konnten, war die Auszahlung lediglich der Hälfte der eingezahlten Rentenversicherungsbeiträge verbunden (Arbeitnehmeranteil ${ }^{16}$ ). Dadurch hat man zugleich eine langfristige Entlastung des deutschen Rentenversicherungsfonds (Fortfall künftiger Risiken beziehungsweise Rentenlasten) erreichen wollen und auch bewirkt. Die Änderung des Rentenversicherungsrechts erfolgte somit nicht nur zu ausländerpolitischen (Ausgrenzung vor allem von Türken und Portugiesen vom deutschen Arbeitsmarkt), sondern auch zu finanzpolitischen Zwecken (gleichzeitige Entlastung der Arbeitslosen- und der Rentenversicherung sowie der kommunalen Sozialhilfehaushalte).

Rechtsförmiger Ausdruck dieser Zwecksetzung war die Koppelung von Rückkehrprämien (als kapitalisierte Arbeitslosenunterstützungsleistung) und erleichterter rentenrechtlicher Anspruchsgewährung (vorzeitig kapitalisierte Rente) mit der aufenthaltsrechtlichen Ausgrenzung der Ausländer (dauernder Ausschluß vom Aufenthaltsrecht für Rückkehrer'7). Gerade der für die Zeit der Geltung des Rückkehrförderungsgesetzes vorgesehene Wegfall der sonst bei der Beitragserstattung nach $\$$ I 303 RVO üblichen Wartefrist von zwei Jahren demonstriert die Preisgabe einer sozialen Schutzfrist zugunsten einer schnellen Ausgrenzung von Ausländern aus dem deutschen Arbeitsmarkt. Insofern hat man das Sozialrecht (Einräumung einer sozialen Schutzfunktion der Wartefrist bei der Geltendmachung der Beitragserstattung) zugunsten eines ausländerpolitischen Zwecks (Entlastung des deutschen Arbeitsmarktes von ausländischen Arbeitnehmern) durch zeitweise Außerkraftsetzung dieser sozialrechtlichen Schutznorm eingesetzt.

\section{Einschränkungen der Sozialhilfe bei Flüchtlingen}

Das dritte und jüngste Beispiel betrifft sogar eine spezifische Gruppe innerhalb der hier vorgestellten Ausländergruppe von Drittstaatern: die Asylbewerber und die sog. de-facto-Flüchtlinge. In diesem Bereich sind arbeitsförderungs- und sozialhil-

14 Zum Zusammenhang von Rückkehrförderung und Bestragserstattung sıehe K. Sievekıng, Die Erstattung von Rentenversıcherungsbeıträgen an Ausländer, Baden-Baden 1988, S. I $7 \mathrm{ff}$.

1s Siehe dazu die Antwort der Bundesregierung auf die Große Anfrage der Fraktion der SPD, BT-Drs. 10/ ${ }_{4} 623$, S. $4:$ :..., auf der Grundlage des Rückkehrförderungsgesetzes wurde der Arbeitsmarkt durch die Rückkehr von rund 140000 ausländischen Arbeitnehmern entlastet $\%$. Aufschlußreich sind auch die Antworten der Bundesregierung auf die Kleıne Anfrage der Fraktıon DIE GRÜNEN, BT-Drs. 10/5432. Welche Bedeutung die Bettragserstattung für die Ausgrenzung insbesondere der türkıschen Arbettnehmer vom deutschen Arbeitsmarkt gehabt hat, läßt sıch an den Antragszahlen und Erstattungsbeträgen gerade im Zeitraum $1983 / 84$ genauer nachweisen; siehe dazu die zahlreichen statistischen Belege beı Sieveking (Fn $\left.1_{4}\right)$, S. $26 \mathrm{ff}$.

I6 Zur Problematik der hälftugen Beıtragserstattung sıehe Sievekıng (Fn 14), S. $88 \mathrm{ff}$.

17 Es war vorhersehbar, daß nach geraumer Zeıt in der Öffentlichkett Bedenken gegen die rıgorosen aufenthaltsrechtlichen Folgen insbesondere für ausländische Jugendliche laut wurden, die zu (nachträglich korrıgierenden) gesetzgeberıschen Aktıvitäten und Änderungen ım Bereıch der Verwaltungsvorschriften der Bundesländer führten, vgl. K. Sievekıng, Das Recht auf Wiedereınreıse für rückkehrende Ausländer, InfAuslR I 988, S. $241 \mathrm{ff}$. 
ferechtliche Normen mit ausländerpolitischen $Z$ wecksetzungen untereinander verkoppelt: Zum einen geht es um das inzwischen auf $\zeta$ Jahre erweiterte Arbeitsverbot für Asylbewerber nach $\S_{19}$ Abs. 1 Satz 2 AFG $^{18}$ bei gleichzeitig möglicher Arbeitsverpflichtung zu gemeinnütziger und zusätzlicher Arbeit nach $\{ }_{19} \mathrm{BSHG}^{19}$. Zum anderen ist der (bislang allerdings) gescheiterte Versuch zu erwähnen, ein eigenes »Asyl-Sozialhilferecht« zu schaffen ${ }^{20}$. Schon jetzt müssen Asylbewerber nach $\$ 120$ Absatz 2 BSHG - anders als Staatsangehörige von Staaten, die nicht Mitgliedstaaten der EG oder des Europarates sind - mit der Kürzung des sonst gewährten Existenzminimums »auf das zum Lebensunterhalt Unerläßliche« rechnen; sie werden auch durch besondere Leistungsarten (z. B. Wertgutscheine) gesondert behandelt $^{21}$. Die Leistungseinschränkungen, teilweise sogar Leistungsverweigerungen gegenüber Flüchtlingen können als Beleg für eine Zweckentfremdung des Sozialhilferechts für ausländer- und asylpolitische Zielsetzungen dienen ${ }^{22}$. Selbst die Rechtsprechung ist sich nicht genau im klaren darüber, ob $\rrbracket_{12}$ Abs. 2 BSHG als ausländerrechtliche oder als sozialhilferechtliche Vorschrift einzuordnen ist ${ }^{23}$.

Obwohl es formell kein eigenes Asyl-Sozialhilferecht gibt, hat sich eine eigene Rechtsform der Verwaltung und Kostentragung von Aufwendungen für die Asylbewerber entwickelt: Es existiert ein vielfältig verwobenes System von Gesetzen und Verwaltungsvorschriften/-richtlinien auf der Ebene der Bundesländer, mit dessen Hilfe die Erstattung von Aufwendungen für die Unterbringung, Versorgung und Betreuung von Asylbewerbern zwischen den Bundesländern und den ihnen zugehörigen Gemeinden reguliert wird ${ }^{24}$. Das Fehlen eines der Kompetenz des Bundes unterliegenden Asyl-Sozialhilfegesetzes wird durch den novellierten $\$ I20 BSHG und ein bereits vorhandenes System von Sonderrecht auf der Ebene der Bundesländer faktisch kompensiert und durch ein entsprechendes Verwaltungsvollzugssy-

18 Näheres beı $M$. Wollenschläger/U. Becker, Das Gesetz zur Änderung asylverfahrensrechtlicher, arbeitserlaubnisrechtlicher und ausländerrechtlicher Vorschriften, ZAR 1987 , S. 5 I ff., 63 f.

19 Vgl. E.Köchling, Die Verpflichtung von Asylbewerbern zu gemeınnützıger Arbeıt, Blätter der Wohlfahrtspflege 1985 , S. I I ff. und B. Schulte/P. Trenk-Hinterberger, Sozıalhilfe. Eine Einführung, 2. Auflage, Heidelberg 1986, S. 198.

20 Siehe dazu Schulte/Trenk-Hinterberger (Fn 19), S. 453; U.Krahmer, Verfassungs- und sozialrechtliche Einwände gegen die geplante Herausnahme der Asylbewerber aus dem Kreıs der Sozıalhilfeberechtıgten sowie zur geplanten pauschalen Absenkung entsprechender Leistungen in einem Sondergesetz, Zettschrift für das Fürsorgewesen 1988, S. $29 \mathrm{ff}$.; M. Zuleeg, Zur Ausgliederung der Sozialhilfeleistungen an Asylbewerber und andere Ausländergruppen aus dem Bundessozıalhilfegesetz, ZDWF-Schriftenreihe Nr. 28, Bonn Juli 1988. Der Deutsche Verein für öffentliche und private Fürsorge hatte schon 1982 die These vertreten und gefordert, eın eigenständiges Asylsozıalhilfegesetz als Bundesgesetz zu verabschieden, vgl. Thesen des Deutschen Vereins zur Frage der Sicherstellung des Lebensunterhalts für asylsuchende Ausländer, NDV 1982, S. 246.

2I Zu den Sonderregelungen innerhalb von $\$ 120$ Absatz 2 BSHG siehe ausführlich $M$. Korde/U. BergerDelhey, Sozialhilfe für Ausländer, ZfSH/SGB 1987, S. $393 \mathrm{ff}$; B. Huber, Asylbewerber im Sozıalhilferecht. Ausgewählte Probleme der sozıalhilferechtlichen Stellung von Asylbewerbern, Nachrichtendienst des Deutschen Vereins für öffentliche und private Fürsorge (NVD) 1988, S. 2 I Iff. Angesıchts der in $\$ 120$ Absatz 2 BSHG eıngeräumten Ermessensspielräume muß man beı der Beurteilung der tatsächlichen Wirkungen dieser Regelungen auch die - wie die Erfahrung zeigt - unterschiedliche Handhabung dieser Ermessensspıelräume mıtberücksıchtıgen, die sıch in den Richtlinien der Bundesländer niederschlagen. Siehe etwa: Aufnahme und Unterbringung der Asylbewerber in Bayern - Januar 1988 -, hrsg. vom Bayersschen Staatsministerıum für Arbeit und Sozıalordnung, S. $57 \mathrm{ff}$., $63 \mathrm{ff}$. Aus welchen Gründen beispielsweise Krankenbehandlungen unterschiedlich gewährt werden, ist nıcht recht nachvollzıehbar; denn Krankheiten sind doch behandlungsbedürftig unabhängıg davon, welche Staatsangehörıgkeıt die betroffenen Menschen haben.

22 So auch B. Huber, Probleme der de-facto-Flüchtlinge bes der Erlangung von Sozialhilfelesstungen, in: M. Kametzki/H. Thomä-Venske (Hrsg.), Schutz für De-Facto-Flüchtlinge, Hamburg 1988, S. 165ff. (17I). Siehe auch J. Lang, Auswırkungen der wflankıerenden Maßnahmen « auf die sozıale Situation von Asylbewerbern in der Bundesrepublik Deutschland, Blätter der Wohlfahrtspflege I988, S. 28 I ff.

23 Vgl. BVerwGE 71, 139 (148).

24 Siehe z. B. R. Kraus, Möglichkeıten und Grenzen sozıaler Hilfen für Asylbewerber - aufgezeıgt am Beispiel Hessen, ZAR 1987 , S. $16 \mathrm{ff}$. 
stem $^{2 s}$ flankiert. Dieser Befund legt die Vermutung nahe, daß eine parlamentarische Behandlung dieses Themas möglicherweise zu unerwünschter Publizität und Aufmerksamkeit geführt hätte. Das Für und Wider zu diskutieren und damit die gegebenen Tatsachen bekannt zu machen, hätte möglicherweise bestimmte Praktiken bei der Behandlung von Asylsuchenden einer breiteren Öffentlichkeit erkennbar gemacht und - nach entsprechender öffentlicher Mißbilligung - verhindern helfen können. Es ist allerdings nicht ganz auszuschließen, daß mit der parlamentarischen Behandlung eine Emotionalisierung des zugrundeliegenden Sachyerhalts bewirkt worden wäre, die angesichts der verbreiteten Ausländerfeindlichkeit zu noch weitergehenden einschränkenden Maßnahmen gegenüber Asylsuchenden hätten führen können ${ }^{26}$. Unter demokratischen Aspekten ist jedoch daran zu erinnern, daß derartige Konflikte öffentlich auszutragen sind - gerade wegen ihrer gesellschaftspolitischen und internationalen Dimension.

\section{Rechtspolitische Folgerungen}

\section{S.I Zur Rolle von Gesetzgebung, Rechtsprechung und Verwaltung}

Die aufgezeigten Beispiele zeigen, daß das Sozialrecht für ausländerpolitische,

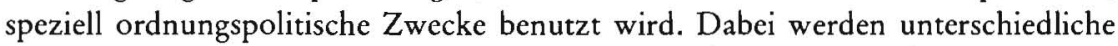
Typen regulativer Politik eingesetzt (Ressource Geld; Ge- und Verbote in Verbindung mit Sanktionen). Rechtsprechung, Gesetzgebung und Verwaltung bedienen sich unterschiedlicher Formen zum Erreichen eines einheitlichen ausländerpolitischen Zwecks: der Ausgrenzung und Abschreckung vor allem derjenigen Ausländer, die aus Staaten außerhalb der EG kommen. Ziel ist es, die Ausländer zur Rückkehr in ihre Heimatländer bezichungsweise zum dortigen Verbleib zu bewegen, um damit das »Inländerprimat ${ }^{27}$ (Deutsche und andere Staatsangehörige von EG-Mitgliedstaaten) auf dem Arbeitsmarkt durchzusetzen. Angesichts der weit verbreiteten »Rückkehrillusion « unter den Migranten ist diese sozialrechtlich abgesicherte Ausländerpolitik rechtspolitisch höchst fragwürdig.

Mit der jeweiligen Instrumentenform sind zugleich bestimmte Verantwortlichkeiten zugeteilt. Die Rechtsprechung übernimmt die Rolle der Gesetzgebung, die ihrerseits ihre Verantwortung nicht wahrnimmt oder wahrnehmen will, weil ein unabweisbares Legitimationsdefizit besteht. Die Gesetzgebung wird dann bemüht, wenn gesellschaftliche Konsense nicht gefährdet erscheinen. Die Verwaltung trägt dem Umstand Rechnung, daß internationale Wanderungsprozesse ebenso wie gesetzliche (Nicht-)Entscheidungen Folgen bewirken, deren Regulierung unabweisbar ist.

Die politische Instrumentalisierung des Sozialrechts für ausländerpolitische Zwecke läßt sich kennzeichnen als »Präventivschutz gegenüber künftigen Sozialkostenbelastungen der gesellschaftlichen Solidarfonds auf Kosten der Ausländer «. Angesichts der hohen Beanspruchung der Sozialversicherungs- und kommunalen Haushalte ist

25 Der Deutsche Städtetag hat hierzu unter dem 13.7. 1987 eine aktualisierte Vorschriftensammlung auf der Ebene der Bundesländer bekanntgegeben, auf die hier pauschal verwiesen wird. Vgl. auch die Vorschriftensammlung von R.Marx, Asylrecht. Band 2: Gesetzessammlung, 4. Aufl. 1984. Siehe auch die Antwort der Bundesregierung auf die Kleıne Anfrage der Fraktion der SPD vom 25. I I. 1987, BT-Drs. II/1 349 .

$26 \mathrm{Ob}$ mit der Handhabung des Asylsozıalhilferechts die These gestützt wird, daß die Massenloyalität des politıschen Systems "selektiv durch Ausschluß von Themen und Betträgen aus der öffentlichen Diskussion « (J. Habermas, Theorıe des kommunikatıven Handelns. Band 2. Zur Kritik der funktıonalistıschen Vernunft, Frankfurt/Main 1981, S. 509) gesichert wırd, müßte ım eınzelnen näher überprüft werden.

27 Vgl. H.Spındler, Die Streıchung von Arbeıtslosenhilfe für Ausländer - gegen die Durchsetzung eınes „Inländerprimats « im Sozıalleıstungsbereıch, InfAusIR 1983, S. $290 \mathrm{ff}$. 
dies erklärbar, aber nicht zu rechtfertigen. So ist zum Beispiel die Schaffung von finanziellen Anreizen zum Verlassen der Bundesrepublik verbunden mit dem Ausscheiden aus den (zwangsweise organisierten) Solidarfonds und dem dauernden Verlust des Aufenthaltsrechts, eine beide Haushaltsbereiche entlastende Maßnahme ${ }^{28}$. Dadurch werden bestehende Ansprüche (Arbeitslosenunterstützungsleistungen, Rentenansprüche, Sozialhilf $\mathrm{e}^{29}$ ) entwertet. Diese Entwicklungen im Bereich der Arbeitslosen- und Rentenversicherung und im Bereich der Flüchtlingssituation dienen offenbar der Abschreckung (gegenüber potentiell Betroffenen) wie auch der staatsinternen Legitimation durch symbolische Entscheidungen. Die sozialrechtliche Komponente der Ausländerpolitik signalisiert damit auch bestehende Überfremdungsängste ${ }^{30}$ der deutschen Politik/Bevölkerung.

Die die Ansprüche von Ausländern vorgeblich erweiternden (z. B. durch Gewährung von Rückkehrhilfe), faktisch aber eingrenzenden Sozialrechtsregulierungen stellen (vermeintliche) gesellschaftliche Konsense her, die infolge von allgemeinen Einbrüchen des Wohlfahrtsstaates (hohe Arbeitslosigkeit; Leistungskürzungen) besonders auf Kosten einer gesellschaftlichen Gruppe erreicht werden. Vereinzelter Widerstand (z. B. bei der Verweigerung von weiteren Zahlungen aus der Arbeitslosenversicherung: instanzgerichtliche Urteile; $z$. B. bei der vorzeitigen Beitragserstattung für rückkehrende türkische Arbeitnehmer: Verfassungsbeschwerde; z. B. beim Versuch, ein eigenes Asylsozialhilferecht zu schaffen: Gutachten für den Hohen Flüchtlingskommissar der Vereinten Nationen) zeigt wenig Wirkung. So bleibt das - soweit bekannt einzige - gegen die Auffassung des Bundessozialgerichts argumentierende und rechtskräftige Instanzurteil ${ }^{31}$ zur Verschlossenheit des Arbeitsmarktes für Ausländer gültig, aber ohne weitere Folgen. Eine Abkehr von der genannten Rechtsprechung ist nach der erneuten Bekräftigung des kritisierten Standpunktes seitens des Bundessozialgerichts ${ }^{32}$ nicht zu erwarten. Die Verfassungsbeschwerde türkischer Arbeitnehmer gegen die auf den Arbeitnehmeranteil begrenzte Beitragserstattung blieb erfolglos ${ }^{33}$, weil - trotz anderslautender Entscheidungen des Bundesverfassungsgerichts zum Eigentumsschutz sozialer Rechtspositionen - der verfassungsrechtliche Schutz der Eigentumsposition im Fall der Beitragserstattung gegenüber Ausländern nicht anerkannt wurde. Schließlich blieb auch den gutachtlichen Äußerungen, die im Auftrag des Hohen Flüchtlingskommissars der Vereinten Nationen in Sachen des Asylsozialhilferechts erstellt wurden, teilweise die Anerkennung versagt ${ }^{34}$.

28 Ergänzend sind auch Förderungsprogramme enzelner Bundesländer mitzuberücksıchtigen, die ergän-

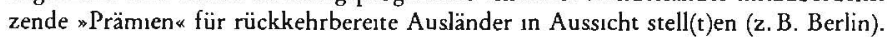

29 Hinzuwersen ist in diesem Zusammenhang auf ausländerspezifische Armut, vgl. Arbetsgruppe ${ }$ Armut und Unterversorgung*, Der tägliche Betrug der Sozıalämter an den Bedürftıgen, Frankfurter Rundschau vom 16.7.1988 (Dokumentation).

30 Siehe dazu K.Sievekıng, Neue Ausländergesetzgebung - deutsche Überfremdungsängste, Demokratıe und Recht 1988, S. $_{4} 12 \mathrm{ff}$.

31 SG Fulda, Urteil vom 28.3. 1985, InfAuslR 1986, S. 16. Das frühere Urteil des SG Gießen vom 19. 1. 1976, KJ 1976, S. 306 mit Anmerkung von B. Huber, wurde - soweıt ersıchtlich - nıcht rechtskräfug. Siehe auch Urteil des SG Duisburg, InfAuslR r 984, S. 323.

32 BSG, Urteil vom 9. 9. 1986, EZAR 432 Nr. I = InfAuslR 1987, 156 mit Anmerkung von K.-J. Bieback, InfAusIR 1986, S. 186. Zur Frage, wann die einjährige Prüfungsfrist, vor deren Ablauf nach der Rechtsprechung des BSG nicht festgestellt werden kann, daß der Arbeitsmarkt verschlossen ist, nicht eingehalten 1st, siehe SG Frankfurt, info also (Informationen zum Arbeitslosenrecht und Sozialhilferecht) $1988, S .112$.

33 Vgl. BVerfG NJW 1988, S. 250 mit Anmerkung von $K$. Sieveking, NJW 1988, S. $246 \mathrm{ff}$.

34 Zur Frage der Verletzung der Menschenwürde hınsıchtlich der Einschränkung der Sozıalhilfe auf das zum Lebensunterhalt Unerläßliche siehe das Gutachten von M. Stollews/G. Schlamelcher, Zur Verfassungsmäßigkeit des $\$ 120$ BSHG, NDV 1985 , S. $309 \mathrm{ff}$., das eine entsprechende Novellierung von $\ 120$ des Bundessozıalhilfegesetzes nıcht bewirken konnte. Zur Zerstörung der Einheit des Sozıalhilferechts und den Gefahren, daß die Betroffenen als Objekt oder gar Störer betrachtet werden, falls em bundeseınheitliches »Asylsozıalhilfegesetz* erlassen würde, siehe Zuleeg (Fn 20). 
Es stellt sich damit immer schärfer die Frage nach der Bewertung eines international durchdrungenen Arbeitsmarktes. Eine solche ist nur auf dem Hintergrund einer zunehmenden Verflechtung der westeuropäischen Nationalstaaten (»Europäischer Binnenmarkt « der EG; Europarat; KSZE) und der dadurch zunehmenden »Diskriminierung der Peripherie « zu diskutieren. Augenfällig wird dies an der rechtlichen und faktischen Behandlung von unterschiedlichen Ausländergruppen innerhalb der Bundesrepublik Deutschland (EG-Staatsangehörige, Drittstaater, Asylsuchende).

\subsection{Zum Zusammenhang von Sozıal- und Ausländerrecht}

Im Lichte der hier beschriebenen Beispiele erscheint die historische Verbindung von Eingriff und Leistung gegenüber Fremden ("gute Polizey") in neuer Form. Offenbar wird die ordnungspolitische Funktion sozialrechtlicher Regulierung als polizeiliche Gefahrenabwehr reinstitutionalisiert. Die als rechtsstaatliche Errungenschaft geltende Ausdifferenzierung von Sozialrecht aus dem alten Polizeyrecht erscheint durch erneute Verpolizeilichung des Sozialrechts teilweise wieder aufgehoben. Dieser (Rück-)Entwicklungsschritt zeigt sich an der Koppelung sozialer und polizeilicher Zwecke im Sozialrecht. Die Besonderheit dieser Entwicklung läßt sich dadurch kennzeichnen, daß das Sozialrecht Polizeiaufgaben ("Gefahrenabwehr«) übernimmt, ohne daß der (ausländer-)polizeiliche Grund ("Überlastung und Überfremdung (?) des deutschen Arbeitsmarktes«) überhaupt ins Spiel gebracht wird. Vielmehr spricht man beispielsweise von einer sozialen Wohltat (Rückkehrförderung), während Ausgrenzung der Adressaten vom deutschen Arbeitsmarkt gemeint ist. Die deklarierten Ziele weichen von den intendierten Zielen ab.

Mit dieser Verknüpfung von ausländer- und sozialpolitischen Zwecken entfernt sich das Sozialrecht von seiner genuinen Funktion, defizitäre Lagen von Individuen abzubauen oder auszugleichen; der durch Eintritt von Risiken (Krankheit, Unfall, Alter, Arbeitslosigkeit, Armut u. a.) erforderliche Ausgleich (Lohnersatz, Krankenbehandlung, Grundsicherung elementarer Lebensbedürfnisse u.a.m.) dient nicht mehr allein der Abfederung sozialer Risiken durch Geld-, Sach- und Dienstleistungen ${ }^{35}$, sondern auch der - nach dem (vermeintlich konsentierten) Gesellschaftsverständnis so definierten - Gefahrenabwehr. Dabei erscheint der längerfristige Aufenthalt von Ausländern als Störung des deutschen Arbeitsmarktes wie auch des gesellschaftlichen Zusammenlebens. Auch der Wechsel der Leistungsform gegenüber einer bestimmten Gruppe von Hilfeempfängern (Übergang von Geld- zu Sachleistungen bei Flüchtlingen) verstärkt diesen Eindruck. Damit übernimmt das Sozialrecht faktisch Aufgaben, für die es an hinreichender Legitimation fehlt.

\subsection{Legitumationsdefizut des Sozialrechts als Gefabrenabwebr}

Im Rahmen der hier genannten Beispiele lassen sich folgende Legitimationsdefizite des Sozialrechts feststellen:

1. Die Rechtsprechung des Bundessozialgerichts zur Verschlossenheit des Arbeitsmarktes beı Ausländern findet keinerlei Stütze in den Rechtsnormen des Arbeitsförderungsgesetzes.

35 Selbst im Wechsel von Lesstungsarten ( $\oint_{\text {II }} S_{\text {SGB }}$ ) zeigen sıch Instrumentalisıerungen, so z. B. beım Übergang von einer Geld- zu einer Sachleistung im Falle der Asylbewerber. Dies verdeutlicht die beabsichtıgten Abschreckungsabsıchten. Zur Flüchtlingspolitik aus bundesrepublikanıscher Sicht sıehe: Flüchtlinge und Asylsuchende in unserem Land, Hrsg. Kirchenamt der Evangelischen Kirche in Deutschland (EKD), Hannover 1986 (EKD Texte I6); H. Rittstteg, Asylrecht und Flüchelingspolitik, ZRP 1986, S.92ff.; außerdem die Reaktıon des Bundestages auf eine Entschließung des Europäischen Parlaments, vgl. Die Woche im Bundestag Nr. $17 / 87$ vom 19. I1. 1987, S. 5; aus der Sicht der EG stehe den Bericht des Abgeordneten Vetter zu den Fragen des Asylrechts an das Europäische Parlament, DOK A2-227/86/B vom 23.2. 1987 . 
Insofern weicht sie von einem Grundsatz rechtsstaatlicher Rechtsprechungstätigkeit, nämlich der Bindung an das Gesetz, ab.

2. Die Rückkehrförderungspolitik durch Prämienzahlung einerseits und verkürzte Beitragserstattung im Rahmen der Rentenversicherung andererseits greift in persönliche Lebensschicksale ein und verkürzt den in sonstigen Fällen gewährten Eigentumsschutz von Rentenanwartschaften erheblich.

3. Die pauschale Schlechterstellung von Asylsuchenden und ihnen gleichgestellten Personen bedeutet den Verstoß gegen das Grundrecht der Unantastbarkeit der Menschenwürde, gegen den Gleichheitsgrundsatz, das Asylgrundrecht sowie die Grundsätze des sozialen Rechtsstaates.

Die sichtbare, allmähliche Aushöhlung von Grundrechten und überkommenen Rechtsgrundsätzen widerspricht der "interkulturellen Verantwortlichkeit ${ }^{36}$ des deutschen Sozialrechtsgesetzgebers und der diese Gesetze vollziehenden Verwaltung im Sinne des Rechts- und Sozialstaatsprinzips. Denn aufgrund der früheren Anwerbevereinbarungen und den international entwickelten Schutzstandards (Grundsatz der Nichtdiskriminierung) bestehen Schutzpflichten gegenüber den ausländischen Arbeitnehmern, z.B. auch hinsichtlich des Erhalts ${ }^{37}$ ihrer durch Arbeit erworbenen Rentenanwartschaften.

Die Einschränkung der Sozialhilfeleistungen gegenüber Asylbewerbern widerspricht dem Gebot der Garantie eines menschenwürdigen Daseins; denn durch unzuträgliche Lebensbedingungen (Arbeitsverbot, Lebensunterhalt durch Wertgutscheinverteilung) wird ein Abwanderungsdruck gefördert, der die Rechte der Ausländer einschränkt und letztlich das Asylgrundrecht nach Art. I6 GG auszuhöhlen droht. Eine Behandlung von Asylsuchenden, die ausschließlich zum Zwecke der Abschreckung anderer erfolgt, mißachtet den sozialen Wert- und Achtungsanspruch von Menschen und verstößt damit gegen Art. I Abs. I GG ${ }^{38}$. Darüberhinaus verstößt diese Regelung wegen der Differenzierung nach Ausländern (Herkunft, Rechtsstatus) und der damit vorgenommenen Abweichung vom reinen Aufenthaltsprinzip gegen Art. 3 Absatz $1 \mathrm{GG}^{39}$. Mit dem Fehlen nicht diskriminierender Lebensbedingungen wird schließlich auch die menschenrechtliche Idee des politischen Asyls in Frage gestellt. Denn aus ihrem Heimatstaat Ausgestoßene sollen für die Dauer des Asyls Aufnahme in eine andere politische Gemeinschaft finden. Diese Betrachtungsweise ${ }^{40}$ steht einer rechtlichen Differenzierung zwischen Staatsangehörigen und Fremden entgegen.

\section{Schlußbetrachtung}

Die in den vorangegangenen Ausführungen sichtbar gewordene teilweise Inkorporation polizeilicher $Z$ wecke in das Sozialrecht erfolgt - das zeigen die hier erwähnten Beispiele - jeweils gegenüber einer besonderen Gruppe von Ausländern, nämlich einer "Minderheit innerhalb einer Minderheit«. Damit wird nicht nur eine weitere Variante einer besonderen Art Sonderrecht innerhalb eines sowieso beste-

36 Ausführlich zur »Interkulturellen Verantwortlichkeit«K. Sievekıng (Fn 14), S. 143 ff.

37 Damit soll nıcht behauptet werden, daß die bıslang verwehrte angemessene Beitragserstattung als solche eine sozıale Wohltat 1st. Solange dieses Institut ene internatıonal-sozıalrechtliche Legitımation findet, bleibt eine Kritik auch an ihrer tatsächlichen Durchsetzungsform legitım.

$38 \mathrm{Vgl}$. Stollets/Schlamelcher (Fn 34), NDV 1985 , S. 313 mit wetteren Belegen in Fußnote 58 . Anderer Ansıcht 1st hier die Rechtsprechung, vgl. OVG Berlin, NDV 1987, S. 233. Zurückhaltender äußert sich das Bundesverwaltungsgericht (BVerwGE 71,139 ), das immerhın eıne Einzelfallprüfung für unumgänglich hält - Im Gegensatz zu pauschalen Kürzungen -, ohne allerdings ım Grundsatz die verfassungsrechtlichen Bedenken zu teilen; ebenso jetzt VG Würzburg, NJW 1988, S. 3110.

39 Ausführlich zum ganzen Stollets/Schlamelcher (Fn 34). Im Ergebnis ebenso Huber (Fn 22), S. 172; stehe auch Schulte/Trenk-Hinterberger (Fn 19), S. 45 if.

40 Vgl. G. Frankenberg, Politısches Asyl - ein Menschenrecht?, K] 1987, S. 17ff., $29 \mathrm{ff.}$ 
henden Minderheitenrechts geschaffen. Dieses spezielle Minderheitenrecht gegenüber einer bestimmten Gruppe von Fremden kann auch als eine Art "Ausnahmerecht « (im doppelten Sinn) verstanden werden; diese Kennzeichnung ist insofern berechtigt, als - wic gezeigt - nicht nur eine Sonderbehandlung spezifischer Gruppen von Ausländern erfolgt, sondern auch einige grundlegende Rechtsprinzipien des allgemein geltenden Rechtssystems partiell außer Kraft gesetzt sind. Hier zeigen sich Gefahren, die mit der übermäßigen Vermehrung von Sozialrecht und der Interdependenz von Sozialrecht und Ausländerrecht zusammenhängen. Insofern können die beschriebenen Phänomene auch als Beispiel für die Gefährdung von Klarheit und Sicherheit des Rechts gelten. Zacher hat im Zusammenhang mit der Verrechtlichungstendenz im Bereich des Sozialrechts davon gesprochen, daß eine äußerste Gefahr darin bestünde, »daß Recht selbst für Erwartungen in Anspruch genommen wird, welche die Sozialpolitik nicht erfüllen kann«. Vor dem Hintergrund der vorangegangenen Ausführungen bleibt festzustellen, daß diese Aussage nicht nur auf einzelne Bürger, sondern offenbar auch auf den Staat zutrifft.

Als Ursache dieser Entwicklung sind - ohne daß hier näher darauf eingegangen werden kann - die von der Politik wesentlich mitgetragene Internationalisierung des nationalen Arbeitsmarktes (verstärkte Anwerbevereinbarungen nach 1961), nationale Sonderentwicklungen (Eingliederung ehemaliger Deutscher - Aussiedler), das wachsende Nord-Süd-Gefälle (Verarmung der Länder der Dritten Welt) und die als Folge der Technikentwicklung seit Anfang der 7oer Jahre - wachsende Arbeitslosigkeit mit ihren Folgen für die gesellschaftlichen Kämpfe um Arbeitsplätze zu nennen. Angesichts der hierauf beruhenden Entwicklungstendenzen verbleiben der Rechtspolitik wichtige Aufgaben, um eine weitere Verpolizeilichung des Sozialrechts und seine zweckfremde Instrumentalisierung zu verhindern.

\section{Dieter Hart \\ Rechtspolitik und Gentechnologie}

Bestehende Regelungen, Regelungsvorschläge der EG-Kommission und der Bundesregierung.*

\section{Vorbemerkung}

Ich beschäftige mich mit der rechtlichen Regulierung der gentechnologischen Forschung, Entwicklung und Produktion, und zwar insbesondere unter dem Aspekt ihrer Sicherheitserfordernisse. Der zweite Abschnitt des Kommentars gilt den sog. Sicherbeitsrichtlinien des BMFT zum Schutz vor Gefahren durch in-vitro neukombinierte Nukleinsäuren. Die Richtlinien regeln Sicherheitsanforderungen für Forschungs- und Entwicklungsarbeiten sowie für die Produktion. Der dritte

\footnotetext{
Der Kommentar basiert auf enem Vortrag, den $1 \mathrm{ch}$ im Oktober 1988 auf Einladung der SPD-Bürgerschaftsfraktion in Bremen gehalten habe. Die Vortragsform wurde weitgehend beibehalten, auf Fußnoten verzichtet; als Literaturhinweis nur: Berıcht der Enquete-Kommission des Io. Deutschen Bundestages, Chancen und Risiken der Gentechnologie, 1987; Damm/Hart, Regulierung riskanter Technologien, KritV 1987, 183-216 und die Betträge zur Gentechnologıe in Heft I1/88 Bundesgesundhertsblatt.
} 